\section{Vibrations as a Cause of Disintegration in Liquid Sheets}

DurING photographic investigations into the disintegration of liquids by means of single-fluid pressure-atomizers, it was established that the liquid first forms a thin sheet which disintegrates well away from the mouth of the nozzle. The modes of disintegration of these sheets are influenced by the properties of the liquid, the nozzle, and the surrounding atmosphere. In certain circumstances, holes are formed in the sheet which are observed to expand rapidly as they travel away from the nozzle. This process is illustrated by a high-speed photograph of a sheet of mercury as it leaves a single-hole fan-spray nozzle (Fig. 1).

It has been established that holes in the sheet can be caused by the presence of non-wettable particles in the liquid or by certain turbulence characteristics in the nozzle, as expressed by an appropriate Reynolds's number. Investigations into the latter cause reveal a phenomenon which may give a clue to the flow mechanism in all types of nozzles traversed by compressible or incompressible fluids. Vibrations which give rise to the noise phenomenon in gaseous jets are thought to be set up by the flow of the gases through the nozzle. These periodic disturbances have for some time been the subject of observation, experiment and mathematical analysis. With liquid jets there is much evidence of turbulent, unsteady flow behind the nozzle being a primary cause of disintegration, but no experimental confirmation of periodic vibrations in liquid jets has yet been forthcoming.

Observations on sheets of liquid such as the above show random centres of disturbance which, as they travel away from the nozzle mouth, eventually break the film and result in holes. Fig. 2 shows a section of a sheet of mercury illuminated obliquely to disclose the disturbances on its surface.

A mathematical analysis of these random disturbances shows that their origin is inside the nozzle

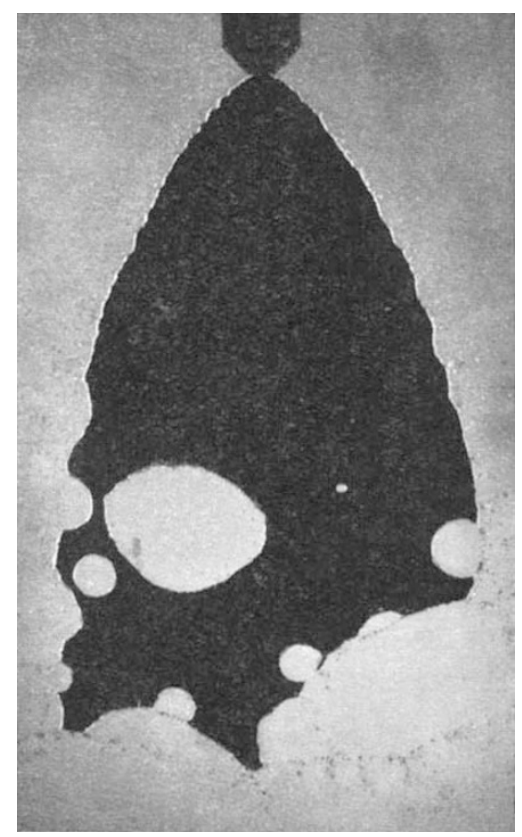

Fig. 1

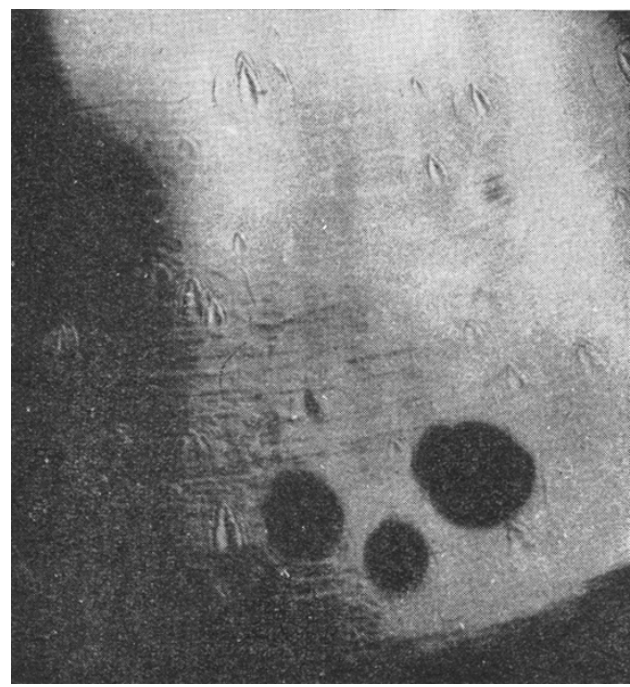

Fig. 2

just behind the mouth, and that the frequency of the observed ripples in the sheet is of the order of $10 \mathrm{kc} . / \mathrm{s}$. Systematic work may reveal the cause of these disturbances and link them to possible vibrational phenomena inside the nozzle.
R. P. Fraser
N. DombrowskI
P. EISENKLAM

Jet Research Laboratory,

Department of Chemical Engineering and Applied Chemistry,

Imperial College of Science and Technology, London, S.W.7. Nov. 17.

\section{High-Voltage Arcs in Argon-Nitrogen Mixtures}

IT has been known for some time that unusual discharges can be maintained in the gaseous filling (argon-nitrogen, 99.5 per cent, 0.5 per cent at about $600 \mathrm{~mm}$. pressure) of commercial incandescent lamps ${ }^{1}$. Recent studies of high-voltage arcs $(1,000-2,000 \mathrm{~V}$., 0-50 m.amp., 60 c./s.) struck in the gaseous filling between the filament support leads of such lamps have been reported ${ }^{2}$. When running the arc at low currents (0-5 m.amp.), it appeared as a yellow-brown 'flame-like' glow, which had a distinct life-time of luminosity of the order of 1 sec. after the power had been switched off. As previously reported, the spectrum of this glow, photographed on Kodak $I-N$ plates between 3500 and $9000 \mathrm{~A}$., showed only the following bands:

\footnotetext{
$\mathrm{N}_{2}$ First positive system : $\quad \begin{aligned} & (1,0),(2,1),(2,0),(3,1),(3,2)[(4,2),(5,3), \\ & \text { faint }]\end{aligned}$

$N_{2}$ Second positive system : $(0,1),(0,2),(0,3),(0,4),(1,3),(1,4),(1,5)$
}

This communication directs attention to probable excitation mechanisms involving vibrational levels of the $X^{1} \Sigma_{g}^{+}$state of $\mathrm{N}_{2}$, which now seem superior to those discussed previously ${ }^{2}$ in the explanation of this unusually low vibrational excitation of the $C^{3} \mathrm{II}_{u}$ and $B^{3} \Pi_{g}$ states of $\mathrm{N}_{2}$ exhibited in this source.

The combined phenomena of unusuelly low vibra. tional excitation of the $C^{3} I_{u}$ and $B^{3} \Pi_{g}$ states, and 